\title{
Advances in hepatocyte transplantation: a myth becomes reality
}

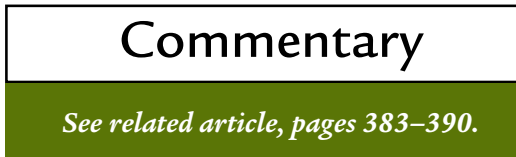

\author{
Linda A. Lee
}

Division of Gastroenterology, The Johns Hopkins University School of Medicine, 720 Rutland Avenue, Ross 1012, Baltimore, Maryland 21205, USA. Phone: (410) 614-1412; Fax: (410) 955-0185; E-mail: 1lee@welch.jhu.edu.

J. Clin. Invest. 108:367-369 (2001). DOI:10.1172/JCI200113650.

The proliferative capacity of the liver is vividly captured in the Greek myth of Prometheus. Having stolen fire and tricked Zeus, Prometheus was chained to a rock and sentenced to have his liver eaten each day by an eagle. Because his liver re-grew nightly, he could endure the same punishment endlessly. Although the myth exaggerates the rate at which normal liver mass can be reconstituted, this organ indeed possesses a remarkable ability to regenerate following partial resection until it attains its original size.

Increasing the rate of liver regeneration to Promethean levels would offer intriguing therapeutic possibilities, especially as the mechanisms that regulate the normal process are becoming better understood. The study by Karnezis et al. in this issue of the JCI (1) suggests that a hepatocyte with enhanced growth potential may not be too fanciful, particularly with the technological advances in genetic engineering that we have witnessed this past decade.

\section{Alternatives to orthotopic liver transplantation}

The most effective therapy for acute or chronic hepatic failure is orthotopic liver transplantation (OLT), first performed in humans in 1963. With 4year survival rates that exceed $70 \%$ for most clinical indications (2), OLT still holds the best therapeutic promise for thousands of patients each year. However, OLT is complicated by several issues, chief among them being a widespread deficiency in donor availability. Additional complications of OLT are the need for lifelong use of immunosuppressive drugs and the extraordinary cost of transplantation, which averages $\$ 193,000$ per patient (3).

The shortage of livers has given rise to transplant waiting lists that are several years long, yet it has also stimulated intensive research to identify novel therapies for liver disease. Hepatocyte transplantation, a "novel" therapy that dates back more than 30 years, takes advantage of the tremendous proliferative capacity of the differentiated hepatocyte. Fewer than 1 in 20,000 hepatocytes divides each day, yet in the face of liver injury, hepatocytes bear the brunt of repopulating the liver. By still-unclear mechanisms that involve cytokines and growth factors, viable differentiated hepatocytes leave their growth-arrested state, become mitotically active, and undergo at least one or two cell divisions until the original liver mass is restored.

Hepatocyte transplantation involves the transfer of normal hepatocytes into diseased liver, by injecting isolated hepatocytes into the spleen or splenic artery or directly into the portal vein. Rhim et al. (4) studied hepatotoxicity and subsequent organ regeneration in a transgenic mouse line engineered to overexpress urokinase plasminogen activator in the liver. They found that administration of $10^{4}$ liver cells could replace more than $80 \%$ of the liver, indicating that donor hepatocytes were capable of at least 12 rounds of cell division (4). Clinical improvement has also been documented in both animal models and human trials of hepatocyte transplantation. Intraperitoneal injections of hepatocytes into rats with fulminant hepatic failure induced by $\mathrm{D}$ galactosamine led to improved survival despite the fact that donor liver cells did not repopulate the liver, even in those rats subjected to removal of two-thirds of their livers (70\% partial hepatectomy), an experimental stimulus for hepatocyte proliferation (5). This finding suggests that hepatocytes can function in ectopic sites with some clinical effectiveness. Clinical and biochemical improvements have been reported in several studies of humans with fulminant hepatic failure treated with intraperitoneal hepatocyte transplantation (6); this thera- py served as a bridge to OLT and in rare cases may have obviated the need for OLT altogether.

Chronic liver diseases that arise from inherited metabolic disorders, such as Crigler-Najjar syndrome type I, may also benefit from hepatocyte transplantation. This congenital disease, which represents an inborn error of bilirubin-UDP glucuronosyltransferase activity and results in unconjugated hyperbilirubinemia, has been treated by transplanting normal hepatocytes through a portal vein catheter. In one case, this treatment led to the survival of donor hepatocytes for up to 11 months and biochemical improvement in a 10-year-old girl (7). Cirrhotic patients who were ineligible for OLT have also undergone hepatocyte transplantation, resulting in improvement in encephalopathy and allowing these patients to undergo portosystemic shunting at a later point (reviewed in ref. 6).

\section{Building a better hepatocyte}

Although these studies indicate that hepatocyte transplantation is potentially useful in the treatment of a diverse spectrum of human liver disease, the limited availability of donor hepatocytes and our inability to culture these cells in sufficient quantities raise the question of whether a hepatocyte that is more adept at liver reconstitution at the time of injury can be devised. Genetic engineering of hepatocytes can already correct specific congenital enzyme deficiencies; ex vivo gene therapy to correct human familial hypercholesterolemia using a retroviral vector has met with success (8). Thus, the technology exists by which gene expression may be altered, allowing one to consider creating a hepatocyte with enhanced growth potential. To create such a hepatocyte, one might target genes that participate in the regulation of the cell cycle, as Karnezis and colleagues have done (1). They 
performed hepatocyte transplantation using hepatocytes isolated from p27 ${ }^{\text {Kip } 1}$ knockout mice to rescue mice with liver failure.

p27 $7^{\text {Kip } 1}$ is an inhibitor of the cell cycle that causes cells to arrest in the G1 phase by inactivating cyclin $\mathrm{E}$ and its associated cyclin-dependent kinase, $\mathrm{CDK} 2$, key regulators of cell proliferation. Inactivation of cyclin E/CDK2 leads to persistent hypophosphorylation of the protein, $\mathrm{pRb}$, such that E2F transcription factors cannot be released to induce expression of genes required for cell proliferation. Several antimitogenic signaling pathways converge on $\mathrm{p} 27^{\mathrm{Kip} 1}$ to cause growth arrest. The role of $\mathrm{p} 27^{\mathrm{Kip} 1}$ in the inhibition of cell proliferation is supported by observations in $\mathrm{p} 27^{\mathrm{Kip} 1}$ knockout mice, which are larger than wild-type animals and have organomegaly in part ascribed to hyperplasia (9-11). The increase in cell numbers of many organs in knockout mice makes p27 Kip1 an attractive target in liver repopulation studies.

The authors have found that cultured p27 Kip1-null hepatocytes isolated from knockout animals have increased DNA synthesis as measured by BrdU incorporation. The clearest evidence of their growth potential is seen in a wellestablished in vivo model of chronic liver disease. The authors used mice deficient in a key enzyme in tyrosine degradation, fumarylacetoacetate hydrolase (FAH). The absence of FAH in these animals mimics hereditary tyrosinemia. To permit normal animal development, animals are given NTBC, a drug that inhibits 4-hydroxyphenylpyruvate dioxygenase and prevents tyrosine degradation. When NTBC is withheld, toxic metabolites of tyrosine degradation rapidly induce liver inflammation and fibrosis; animals die within 4-8 weeks after withdrawal of the drug.

To determine whether hepatocytes

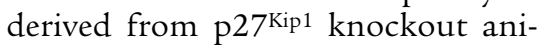
mals possess improved growth potential over wild-type hepatocytes, Karnezis et al. injected $10^{4}$ p27 $7^{\text {Kip } 1 \text {-null }}$ hepatocytes into the spleens of FAH knockout mice after NTBC withdrawal (1). Mice injected with p27 Kip-null hepatocytes survived significantly longer than did mice injected with wild-type cells. Although liver repopulation was variable in identically treated animals, the p27 Kip1 knockout hepatocytes repopulated the liver more extensively than did wild-type hepatocytes. The better survival and repopulation rate of $\mathrm{p} 27^{\mathrm{Kip} 1}$-null hepatocytes could not be attributed to an increase in FAH synthesis or to a decrease in apoptosis.

It is remarkable that $\mathrm{p} 27^{\mathrm{Kip} 1}$ knockout hepatocytes have as profound an effect as they do; although p27 Kip1 knockout mice are prone to gigantism, liver enlargement is not as impressive as the enlargement of other organs, perhaps because $\mathrm{p} 27^{\mathrm{Kip} 1}$ expression is normally lower in the liver than in other tissues. However, this study demonstrates that genetically altered hepatocytes could be superior to wildtype cells in repopulating a damaged liver, even though significant liver repopulation may not be required for prolonged survival, as demonstrated by the one long-term survivor with only $2 \%$ hepatic repopulation in the Karnezis study (1). They found, however, that weight gain correlated with significant liver repopulation rates of $62 \%$ or more.

\section{Future clinical applications and concerns}

These experiments illustrate that transplanted hepatocytes are capable of surviving and organizing in the liver despite ongoing injury in FAH-deficient host hepatocytes. Whether transplantation of $\mathrm{p} 27^{\text {Kip } 1 \text {-null hepatocytes }}$ would be beneficial in the treatment of multiple types of liver disease remains uncertain, particularly in the acute setting. The most common etiologies of fulminant hepatic failure in the US are acetaminophen toxicity and cryptogenic (unknown) causes (12). It is possible that transplanted hepatocytes will be as susceptible as host hepatocytes to drug- or virus-induced injuries. Indeed, Braun et al. (13) found that, even following moderately successful liver repopulation by donor hepatocytes in a mouse model of drug-induced liver failure, survival rates did not improve. These authors concluded that ongoing hepatocyte injury did not allow donor hepatocytes to organize into normal parenchymal architecture (13). Thus, hepatocyte transplantation is unlikely to singularly replace OLT for the treatment of all types of liver failure.

If hepatocyte transplantation is to be a viable therapeutic alternative to OLT, significant liver parenchyma repopu- lation could be required, although the absolute liver mass required to maintain liver function remains unknown. Despite this uncertainty, hepatocyte transplantation has several potential therapeutic advantages over OLT. The cost of hepatocyte transplantation is estimated to be one-tenth the cost of OLT, so multiple hepatocyte transplantations could be performed in the same patient in a cost-effective manner (6). Several patients could be potentially treated from one donor liver, with the present ability to cryopreserve isolated hepatocytes for later use. With the increase in living related donor transplantation, in which a segment of liver from a living individual is transplanted into a patient, the availability of isolated hepatocytes may increase. Methods of transgene delivery in human primary hepatocytes have advanced, making possible autologous transplants and the ability to dispense with concomitant immunosuppressive therapy. Perhaps most attractive is the prospect of being able to genetically manipulate isolated hepatocytes to develop a "super-hepatocyte," capable of enhanced proliferation but less susceptible to apoptosis or ongoing hepatic injury. In addition to targeting genes that regulate the cell cycle, it may be possible to alter critical genes that control protein synthesis and metabolism with the aim of improving hepatocyte function, such that fewer hepatocytes would be needed to do the work of the liver.

Naturally, the same modifications that distinguish such a "super-hepatocyte" could predispose it to cancer formation. Although the authors (1) point out that loss of $\mathrm{p} 27^{\mathrm{Kip} 1}$ has not been associated with hepatic tumor development in knockout mice, other studies indicate that p27 ${ }^{\mathrm{Kip} 1}$ levels in general are higher in hepatocellular carcinoma than in nontumorous tissue and that those tumors with the highest $\mathrm{p} 27^{\mathrm{Kip} 1}$ expression are associated with a better prognosis. Certainly, it is worrisome that $\mathrm{p} 27^{\text {Kip }}$ knockout mice are susceptible to tumor formation in other organs when exposed to carcinogenic stimuli, perhaps because the loss of $\mathrm{p} 27^{\mathrm{Kip} 1}$ predisposes to genetic instability when cells are challenged with carcinogens. The major role of the liver in drug metabolism and detoxification only heightens this concern. Only long- 
term studies in rodents will determine whether increased tumor susceptibility is conferred with loss of p27 $7^{\mathrm{Kip} 1}$ in hepatocytes. The lifespan of $\mathrm{p} 27^{\mathrm{Kip} 1}$ knockout hepatocytes in the liver must be determined in order to know how much weight this issue should be given; other studies have documented the persistence of transplanted hepatocytes for over 11 months. If tumorigenesis remains a major concern, it may be possible to design hepatocytes engineered to carry suicide genes, which, when activated, cause transplanted hepatocytes to "self-destruct" after the full recovery of the patient has been ensured. In the nearer term, short-lived pharmacologic agents that block p $27^{\mathrm{Kip} 1}$ function or decrease its half-life in transplanted cells may serve as powerful adjuvants to improve existing transplantation protocols.

Hepatocyte transplantation may become standard therapy in the near future. We now have the technology to conceive of a hepatocyte with enhanced growth potential; this may not be the answer for all types of liver disease, but the concept is intriguing. The rapidly regenerating liver of Prometheus may soon be a reality.

\section{Acknowledgments}

The author thanks E. Emison and C.V. Dang for their critical review of this manuscript.

1. Karnezis, A.N., Dorokhov, M., Grompe, M., and Zhu, L. 2001. Loss of $\mathrm{p}^{27^{\mathrm{Kip} 1}}$ enhances the transplantation efficiency of hepatocytes transferred into diseased livers. J. Clin. Invest. 108:383-390.

2. Keeffe, E.B. 2001. Liver transplantation: current status and novel approaches to liver replacement. Gastroenterology. 120:749-762.

3. Gilbert, J.R., et al. 1999. Evolving trends in liver transplantation: an outcome and charge analysis. Transplantation. 67:246-253.

4. Rhim, J.A., Sandgren, E.P., Degen, J.L., Palmiter, R.D., and Brinster, R.L. 1994. Replacement of diseased mouse liver by hepatic cell transplantation. Science. 263:1149-1152.

5. Makowka, L., et al. 1980. Cellular transplanta- tion in the treatment of experimental hepatic failure. Science. 210:901-903.

6. Strom, S.C., Chowdhury, J.R., and Fox, I.J. 1999 Hepatocyte transplantation for the treatment of human disease. Semin. Liver Dis. 19:39-48.

7. Fox, I.J., et al. 1998. Treatment of the CriglerNajjar syndrome type I with hepatocyte transplantation. N. Engl. J. Med. 338:1422-1426.

8. Grossman, M., et al. 1995. A pilot study of ex vivo gene therapy for homozygous familia hypercholesterolaemia. Nat. Med. 1:1148-1154.

9. Fero, M.L., et al. 1996. A syndrome of multiorgan hyperplasia with features of gigantism, tumorigenesis, and female sterility in p27(Kip1)-deficient mice. Cell. 85:733-744.

10. Kiyokawa, H., et al. 1996. Enhanced growth of mice lacking the cyclin-dependent kinase inhibitor function of p27(Kip1). Cell. 85:721-732.

11. Nakayama, K., et al. 1996. Mice lacking p27(Kip1) display increased body size, multiple organ hyperplasia, retinal dysplasia, and pituitary tumors. Cell. 85:707-720.

12. Ostapowicz, G., and Lee, W.M. 2000. Acute hepatic failure: a Western perspective. J. Gastroenterol. Hepatol. 15:480-488.

13. Braun, K.M., Degen, J.L., and Sandgren, E.P. 2000. Hepatocyte transplantation in a model of toxin-induced liver disease: variable therapeutic effect during replacement of damaged parenchyma by donor cells. Nat. Med. 6:320-326. 